Lints, T. (2010). Coordination issues in modular systems. In 4th Annual IEEE Systems Conference, pages 202-206. IEEE.

T. Lints, "Coordination issues in modular systems," in 4th Annual IEEE Systems Conference, pp. 202-206, IEEE, 2010.

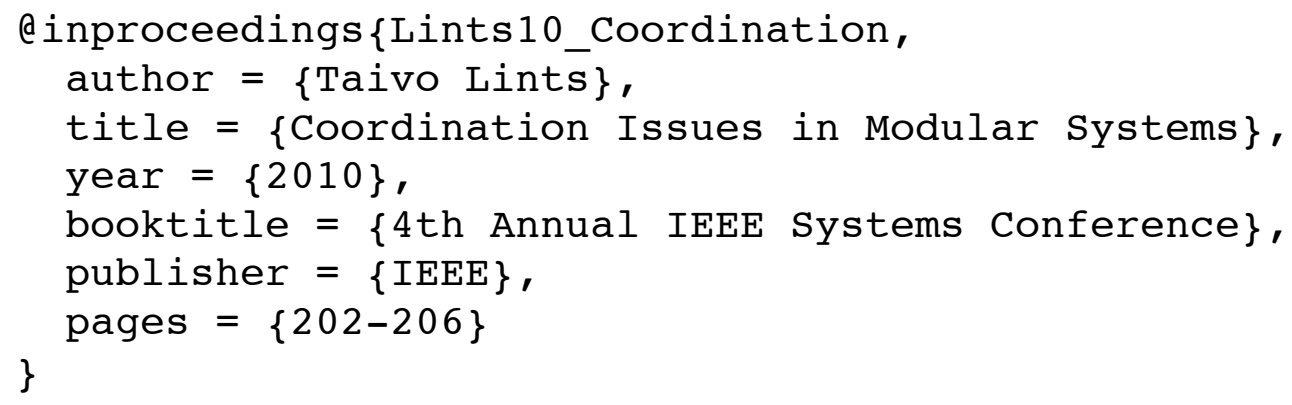




\title{
Coordination Issues in Modular Systems
}

\author{
Taivo Lints \\ Research Laboratory for Proactive Technologies \\ Tallinn University of Technology \\ Ehitajate tee 5, 19086 Tallinn, Estonia \\ Email: taivo@taivo.net
}

\begin{abstract}
In modular systems consisting of partially independent components the application of full central control is usually both undesirable and impossible. But this makes subunit coordination and overall system coherence maintenance difficult. The paper gives an overview of various potential approaches to alleviate that problem, based mostly on human / organizational research literature but encouraging the use of those ideas as inspiration for the design and management of systems of all kinds. The main covered issues are organizational structure, dealing with spontaneously emerged behavior, and supporting component cooperation (teamwork).
\end{abstract}

\section{INTRODUCTION}

In many modular systems, especially the more complex ones (and particularly in systems-of-systems), the modules have a certain level of independence in some aspects of their functioning. This means that often there is no possibility to apply full central control, which actually can provide quite a few benefits for the system and is thus frequently an intentionally sought for property, but at the same time it increases the difficulty of coordinating the activities of subunits and makes it harder to maintain the required level of overall coherence in the system. Possible coordinative actions applicable in such cases include activity pacing, response sequencing, and time and position coordination [1], workload distribution, division of labor and roles, etc., or, as expressed in [2]: "The critical issues in team mission processing are: what should be done, who should do what, with which resources, and when.". Special efforts of coordination may be needed in times of system transformations so as to avoid inconsistent changes by deciding whether, how, when and which components should adapt [3]. If enough coordinative actions are not put into effect properly, the system can easily degrade into an unconstructive mess full of interference, conflicts, undone tasks, and lack of synergy.

Coordination surely requires some kind of functioning communication to be present in the system and is affected by the structure and dynamics of that communication subsystem. This is, however, a large area of research on its own, not uniquely tied to coordination, and thus in the following the underlying

This work was supported in part by Research Laboratory for Proactive Technologies in Tallinn University of Technology, Department of Computer Control in Tallinn University of Technology, Estonian Doctoral School in ICT, Estonian Information Technology Foundation, and Estonian Ministry of Education and Research (grant SF0140113As08). communication channels are assumed to already be in place and functional.

The paper is mostly based on human / organizational research literature, but the intent is to facilitate cross-disciplinary knowledge transfer, i.e., to encourage the use of those ideas as inspiration for the design and management of systems of all kinds.

\section{Organizational Structure}

One of the main characteristics affecting coordination in a modular system is the organizational structure - in what roles the components collaborate with each other and do they have different levels of commanding rights. It is possible to identify several "axes", relevant to organizational structure, along which various systems can be plotted.

The first would be about how much structure there is ranging from none to an extremely structured system. And closely related to that is another axis: the flexibility of the organization - how easily the system can change its level of structuredness (i.e., move along the first axis), or alter the type of that structure, or also modify the command and subordination relationships while staying within one type. Some possible limit cases are discussed in [4]:

At one extreme, a group of disparate, unstructured people and tools can find it greatly difficult to create value. The absence of communication protocols, shared knowledge, compatible tools, and common goals renders the timely and economical accomplishment of goals quite problematic. At the other extreme, a group of rigidly structured people, processes, and tools can also find it challenging to accomplish anything except perhaps an anachronistic goal, and even that with great inefficiency.

Thus, some intermediate balance is the most desirable, as usual. One possibility is suggested by the study of multi-agent systems where one of the core ideas that allows for some structure to exist in the system but keeps it from becoming overly rigid is to have the agents making requests instead of issuing orders. The receivers of the requests can then decide whether and when they are going to fulfill those (but still do comply often enough so that the system does not degenerate into uncooperative solitary entities).

An additional level of flexibility may be attained by making the interactions anonymous and uncoupled, so that the inter- 
action partners need neither to know each other in advance, nor to be active at the same time [5]. This could be achieved by publishing the requests in some medium (ranging from simple informational objects or messages placed into the environment or onto note boards, to sophisticated proactive information systems) and allowing the initiative to fulfill them come from any interested parties. This assumes, of course, that the system is composed of such agents that do engage voluntarily in cooperation. Which provides us with a third possible axis: the alignment of components' goals with those of the system. Here, the range is from totally conflicting goals to fully aligned. Roughly speaking, it would be the same as ranging from extremely malicious (from the system's perspective) subunits through the neutral or disinterested up to the cooperative and (hopefully) synergetic ones. Note, however, that goal alignment does not necessarily imply that the goals of the components are identical to those of the system as a whole. Also, if alignment is defined relatively strictly as a strong enough similarity between the goals (as opposed to the more general way that asks component goals to only support the overall objectives in some way), then high alignment does not necessarily mean high synergy and efficiency in moving towards fulfilling the goals. This derives from the fact that in certain situations and groupings a set of elements with somewhat misaligned goals may actually perform better than one with nearly fully overlapping goals. Examples include some forms of competitive markets and well-utilized diversity.

Another characteristic relevant to organizational structure is the self-sufficiency of the components, ranging from low to high, as this underlies the need for the system to have a well-functioning supportive structure. Self-sufficient components can survive by themselves and thus can do without the organizational support, even though having the latter is still a fully viable option, often indeed the preferred one. At the other extreme, the total lack of self-sufficiency generally demands for some kinds of reliable relationships that provide the missing functionalities or resources. In practice this issue tends to be linked with specialization: the more specialized something is, the more it is inclined to drop all other abilities outside its field of expertise in order to increase the efficiency in that particular skill.

Finally, the classification most associated with the concept of organizational structure, up to the point of being synonymous with it for some, is that of egalitarian vs. hierarchical, which actually can again be taken as a more or less continuous axis from purely horizontal to totally vertical relationships. In an egalitarian system components have more or less equal rights and opportunities, whereas in a hierarchical system there are different levels of authority, lower ranks being expected to obey the orders of higher ones.

A good description of the benefits of egalitarianism, in military context, is given in [6], saying that a decentralized approach "might achieve force agility through drawing on highly parallel and distributed local knowledge to test for the continuing validity of critical assumptions and conditions, and might then develop appreciation of the needs and options for change through collaborative interactions between the key decisionmakers, who are then also responsible for facilitating implementation". While this sounds perfect for an adaptive system, there are unfortunately some drawbacks, too. The most obvious is the high risk of the system to become overly incoherent as there is no central manager. This problem might not occur, however, if the system has appropriate selforganizational properties. But there are still more weak spots: too flat an organization may have a large inertia compared to a hierarchical one, and at the same time short conceptual time horizon matching the time horizon of individual components [6], [7].

Centralization of decisionmaking, on the other hand, "facilitates agile changes to strategy, or course of action as required by the dynamics of the situation, provided adequate means exist to know when a change is called for, and to communicate the change required to deployed forces that are already executing the current course of action" [6]. Also, hierarchical command structures provide "the ability for forces to simultaneously operate at, and adapt across, multiple physical and temporal scales, so that while lower level forces respond to immediate combat pressures, senior commanders can be shaping the longer term battle" [6]. Thus, hierarchical systems can often be faster than egalitarian ones, and better support multi-scale processing where local small viewpoints and farreaching broad perspectives are both present and well balanced. But, surely enough, this comes with a price, again. In a strongly hierarchical organization the decisionmakers are often far removed from the actual fields of action and may thus miss important details of the situation, leading to unfit decisions and commands. Further, as authority is focused in the commander, lower rank elements are often denied the ability to adapt or work around a problem ( [8], as summarized in [9]), which underutilizes the useful capabilities and knowledge present in the subordinates (thereby potentially limiting the total range of system's capabilities) and decreases the system's robustness (losing a commander would likely be highly disruptive and cause a massive drop of fitness). And, in case of deliberate cognitive individuals such as humans, the less involved the subordinates are in the decision making process and the less their interests are taken into account, the less they also feel motivated to do a good job in implementing these decisions. Moreover, it has been observed that in human systems it is the combination of high power distance (the extent to which people accept the unequal distribution of power) and high collectivism (the disposition to prioritize the group over its individual members) that results in the strongest propensity to resist change and thus stifles adaptivity ([10], drawing on [11] and [12]).

To overcome the described limits, it would be a good idea to steer clear of the extremes, take the best features of both egalitarian and strictly hierarchical systems, and try to join them in some hybrid approach. At least three options can be distinguished.

The first possibility is to have a mostly hierarchical system but loosen up the dictate and allow the subordinates a greater 
freedom of decision (while still routing quite a lot of communication vertically through the higher level supervisors). For example, it has been suggested that "The role of higher level headquarters that manage semi-autonomous teams will be to clearly communicate and then police the boundaries of acceptable behaviour, within which autonomous teams have freedom to innovate." [13] which can well be combined with the advice from [6] to make the supervisors facilitate local initiative and adaptation within the context and limits of an agreed framework, as well as to allow for direct command intervention by exception when necessary but avoid too frequent micromanagement.

The second option is to have a mostly egalitarian system where various hierarchies are formed temporarily, based on current needs and on the capabilities and experience of the components. Credibility and legitimacy of leadership should be premised on expertise specific to the task at hand, rather than on the basis of some less related indicators such as hierarchical status, seniority or age that are often used in human groups [14]. Also, in addition to changing the task- and situation-dependent organizational structures sequentially, a complex enough system could have many different hierarchies existing simultaneously, on the same set of nodes, such that there would be distinct command and report chains for the various different roles each component may (simultaneously) be in.

The third alternative is to have a relatively fixed organizational network, but instead of the classical tree arrangement use something more akin to some non-tree kinds of scale-free networks that have a (balanced) mixture of well-connected neighbors and of hierarchical structure.

Which option to choose depends, among other things, on the initial conditions. When starting from a strictly hierarchical system it is most probably the easiest to use the first approach, and from an egalitarian the second. The suitability of the options depends also on component complexity - elements with very limited capabilities might benefit from (or even require) having a well-defined organizational structure in place, whereas complex cognitive agents are better equipped for setting up the appropriate networks on the spot depending on the context and goals.

\section{Dealing with Spontaneously Emerged BEHAVIORS}

One of the issues in assuring sufficiently coordinated and coherent goal-directed behavior is how to deal with various processes that have emerged spontaneously, and often unexpectedly, and that may not behave as supportively with regard to system's goals as desired.

For example, in organizational research it has been observed that spontaneously emerged and survived mechanisms that form the "culture of the organization" create a great deal of organizational inertia to change [7], especially if they have appeared during the formative phases of the system, as noted in [15] (referring to [16]): "norms established early in a group's existence tend to continue even after their value is no longer evident". This, obviously, can have dire consequences for system's adaptivity.

Solving that problem in a deliberate way should usually start with creating the ability to recognize when and which emergent behaviors actually do (or will) conflict with the processes crucial to the fulfillment of the system's major goals, or that are in general troublesome and unproductive. When the conflicts are detected, or possibly already earlier in a preventive manner, the fitness functions / goals of the spontaneously arised mechanisms should be modified so that they would be better aligned with the overall organizational intent [7]. This might be achieved by identifying the points of influence that are most effective for modifying the mechanisms' operation [7] and impacting on those appropriately. In practice, however, it can often be far beyond the system's (or even of the potentially vastly smarter external agents') capabilities to accomplish such a delicate intervention, and much more aggressive measures might be used, up to the expulsion or disintegration of the offending set of modules, if possible to locate and localize. But in most cases, of course, the early prevention would be the preferred solution, requiring less effort, being less disruptive and more effective. As noted earlier, the (potentially unsuitable) behaviors often get fixed already in the first developmental stages of the system, thus the main preventive efforts should be focused on that time (or on the preceding design phase, if exists). Even if such early prevention typically assumes some ability to predict emergent behavior, it can nevertheless be done, in principle, to some extent and for some range of situations. And the less there is known about the system's future environments and goals, the more these precautionary measures should focus on keeping the system adaptive, as opposed to supporting only those mechanisms that seem the most efficient in the system's current context.

\section{SUPPORTING TEAMWORK}

Due to its high practical value a lot of research has been done on facilitating human teamwork. As it is mainly the coordination, implicit or explicit, that distinguishes a team from a mere collection of individuals, the ideas from that research are well worth taking a closer look at here (while keeping in mind that they may well serve as an inspiration for bettering non-human-based systems, too).

The factors and measures affecting teamwork can be roughly divided into two categories: those relevant on the level of an individual, and those on the collective, group level.

\section{A. On the Individual Level}

On the individual level one of the main problems is that being a useful member of a team often requires additional resources and capabilities that allow and support intercomponent interactions. Especially in systems with complex components, "teams can all too easily add to their members' cognitive workload rather than reducing it. This is because any one individual's mental model has to include other team member nodes in his/her definition of the situation." [17], possibly 
including their behavior, intentions, knowledge, mental models, etc. Also, it is often useful or necessary for the components to have knowledge about, and take into account when doing something, team level tasks, goals, rules, organization, and so on, which again calls for additional resources and capabilities for coping with that extra workload. But even in simpler systems more often than not additional special mechanisms are needed in the subunits to enable fruitful interactions.

It is frequently also necessary, again particularly for the systems with more complex individuals, to break the "addiction to perspectives similar to one's own. It is important to learn to listen to others with different points of view" [18], because smooth teamwork typically requires compromises and tradeoffs between the goals and opinions of constituent agents. Additionally, if no single component has the full knowledge of the situation and correct all-encompassing mental models, as is usually the case, it is to be expected that isolated individual decisions might not always be the best, and that listening to others' viewpoints can lead to more fit behavior. So, taking all this into account, the individuals should, as described in [19], resist the urge for closure and certainty, not be seduced by one's own ideas, appreciate that it is much more important to be prepared to be wrong in order to learn than to always be right (and therefore either or both risk-averse or in denial) and conversely, be prepared to 'decriminalize' others being wrong.

Other individual characteristics that can be helpful for good teamwork and that are particularly relevant for cognitive agents but inspirational also when designing simpler components include: self-awareness, social awareness, organizational awareness, interpersonal maturity, critical thinking [9]; cooperativeness, sociability, social intelligence [20]; cultural competence, awareness of differences (between self and others), suitable (i.e., matching with partners) cognitive style and public space [1]; and many more.

\section{B. On the Group Level}

Then there are teamwork-supporting factors and measures that exist only on the group level, i.e., require more than one individual to be present. These include, but are by far not limited to: team motivation and attitudes, experience of the team (as a team, not just the sum of experiences the components may have gathered alone or in other groups), team heterogeneity, team leadership (whether there is a member in the team to whom others look for guidance and who also serves as a role model), distribution and mutual understanding of roles, shared / compatible mental models [20]; and team situational awareness (a shared understanding of a given situation at a given point in time) [21].

With regard to the group level factors - one of the frequent pitfalls in human groups is the assigning and adjustment of roles and responsibilities based on characteristics that are only indirect, and often incorrect, indicators of persons' suitability, such as social / organizational position or age. At least in principle this assignment should be instead based on the appropriate "matching of member resources, skills, abilities, prior knowledge, task information, numbers, etc., to subtask requirements" ( [22], as cited in [1]). In practice, though, the latter approach may lead to complications, too, when people do not get the roles they think they deserve and will therefore underperform or sabotage due to being dissatisfied, so the issue should be handled carefully (via, for example, open honest dialogue, fair sharing of the benefits, etc.).

Another important aforementioned factor in ensuring fluid teamwork - the sharing of information and mental models is also far from trivial. This topic would certainly deserve a detailed integrative study of its own, but to get started, here are a few examples of what should be considered (though not necessarily always implemented) when designing intra-team information sharing:

- The sharing of information about both successes and failures [23, page 66], [18].

- The sharing of information about member resources and constraints, team tasks and goals or mission, environmental characteristics and constraints, and about the assignment of priority among subtasks [1].

- The construction and regular discussion of mental models while at the same time avoiding the danger of harmful groupthink [19].

- The system-wide maintenance of the knowledge about system's components, current status, ultimate capacity, connections with other systems, the extent of its "owned" resources, those it can borrow or lend, and those that can be shared or should be isolated [24].

Other teamwork-supporting methods include:

- The (deliberate) creation of a specific team culture (a "third culture") that is suitable for all participants and supports the team's goals, as opposed to expecting everybody to accept some existing majority culture or just letting it unguidedly arise by itself [25].

- Managing changes through the provision of (new) enabling and supportive infrastructures instead of direct forceful interventions that might irritate the components and cause conflicts [26].

- Recognizing and mitigating potential adverse crosseffects and conflicts between the elements [19].

- Setting shared standards of performance.

- Keeping the size of the group within suitable limits.

Finally, it is advisable to pay attention to the maintenance of safety and trust among the individuals, otherwise the spread of useful (or even any) ideas and information may become strongly inhibited. It is vital for team adaptivity to make sure that the team is safe for interpersonal risk taking: speaking up, offering suggestions, critiques, expertise, advise [21]. Furthermore, too much adaptability can lead to too much uncertainty for the components, which in turn diminishes their trust in each other [27], especially if the network rapport is implicit and informal and therefore builds up slowly [26]. Thus, from this perspective, the speed of adaptations should be kept reasonably low, or, preferably (assuming we are aiming for high adaptivity), explicit mechanisms should be 
put in place that guarantee the preservation of individual safety and trustability throughout adaptational processes or at least provide the components with some reliable indicator of others' trustability (in relevant aspects) so as to avoid everybody distrusting everybody by default, "just in case".

\section{CONCLUSION}

Coordination is an important issue in a majority of (functional) systems that consist of more than one component, and quite a lot of research in various disciplines has been done to understand it better. This paper was based mainly on human / organizational studies' literature and had the intention to gather together some of the useful ideas and suggestions so as to serve as a concise notebook to get some initial ideas and inspiration from when dealing with the analysis, management and synthesis of both social and technical modular systems. But it is certainly very far from being a thorough survey (or, actually, much of a survey at all - it is more like a sampling of such sources that one would not normally know to turn to if starting to look for information about coordination) and a lot of additional practical ideas can be found in the literature of organizational research, as well as of distributed computing, self-organization, logistics, biology, and more.

As of how to ensure the existence of the listed supportive characteristics, capabilities and processes in a system, and which ones to prioritize in which situations, no universally easy solution is likely to exist and the main methods would be the usual design time considerations and later analyses, adjustments, training and, if necessary, replacement of the components individually, or grouped as subsystems, or the system as a whole. But, on the positive side, the effectiveness of these methods can surely be raised by acquiring a better understanding of the concept of coordination (and, more generally, of the working principles of complex adaptive systems) via further research and refinement of the resulting ideas into practically usable guidelines.

\section{ACKNOWLEDGMENT}

Thanks to Leo Mõtus for providing me the possibility to do highly unconstrained research.

\section{REFERENCES}

[1] J. L. Sutton, L. G. Pierce, C. S. Burke, and E. Salas, "Cultural adaptability," in Understanding Adaptability, A Prerequisite for Effective Performance within Complex Environments, C. S. Burke, L. G. Pierce, and E. Salas, Eds. Elsevier, 2006, pp. 143-173.

[2] G. Levchuk, D. Serfaty, and K. R. Pattipati, "Normative design of project-based adaptive organizations," in Understanding Adaptability, A Prerequisite for Effective Performance within Complex Environments, C. S. Burke, L. G. Pierce, and E. Salas, Eds. Elsevier, 2006, pp. 249287.

[3] P. G. Bridges, W.-K. Chen, M. A. Hiltunen, and R. D. Schlichting, "Supporting coordinated adaptation in networked systems," in Proceedings of the Eighth Workshop on Hot Topics in Operating Systems. IEEE Computer Society, 2001.

[4] T. R. Browning, "Program architecture and adaptation," in RAND Corporation Symposium on Complex Systems Engineering, 2007.

[5] M. Mamei, R. Menezes, R. Tolksdorf, and F. Zambonelli, "Case studies for self-organization in computer science," Journal of Systems Architecture, vol. 52, pp. 443-460, 2006.
[6] M. Unewisse and A.-M. Grisogono, "Adaptivity led networked force capability," in International Command and Control Research and Technology Symposium, 12th ICCRTS, 2007.

[7] A.-M. Grisogono, "Co-adaptation," in SPIE Symposium on Microelectronics, MEMS and Nanotechnology, A. Bender, Ed., 2005, pp. 23-37.

[8] G. Klein and L. Pierce, "Adaptive teams," in 6th International Command and Control Research Technology Symposium, 2001.

[9] J. C. Tillson, W. D. Freeman, W. R. Burns, J. Michel, J. A. LeCuyer, R. H. Scales, and D. R. Worley, "Learning to adapt to asymmetric threats," Institute for Defense Analyses, Alexandria, VA, Tech. Rep. D3114, 2005.

[10] J. Giacobbe-Miller, D. Miller, W. Zhang, and V. Victorov, "Country and organizational-level adaptation to foreign workplace ideologies: a comparative study of distributive justice values in China, Russia and the United States," Journal of International Business Studies, vol. 34, pp. 389-406, 2003.

[11] A. Harzing and G. Hofstede, "Planned change in organizations: the influence of national culture," in Research in the Sociology of Organizations: Cross-Cultural Analysis of Organizations, P. Bamberger and M. Erez, Eds. JAI Press, 1996, pp. 297-340.

[12] B. Kirkman and D. Shapiro, "The impact of cultural values on employee resistance to teams: toward a model of globalized self-managing work team effectiveness," Academy of Management Review, vol. 22, no. 3, pp. 730-757, 1997.

[13] A. Ryan, "About the bears and the bees: Adaptive responses to asymmetric warfare," in Proceedings of the Sixth International Conference on Complex Systems, A. Minai, D. Braha, and Y. Bar-Yam, Eds. New England Complex Systems Institute, 2006.

[14] G. L. Harrison, J. L. McKinnon, A. Wu, and C. W. Chow, "Cultural influences on adaptation to fluid workgroups and teams," Journal of International Business Studies, vol. 31, no. 3, pp. 489-505, 2000.

[15] H. Moon, J. R. Hollenbeck, S. E. Humphrey, D. R. Ilgen, B. West, A. P. Ellis, and C. O. L. H. Porter, "Asymmetric adaptability: Dynamic team structures as one-way streets," Academy of Management Journal, vol. 47, no. 5, pp. 681-695, 2004.

[16] K. Bettenhausen and J. K. Murnighan, "The emergence of norms in competitive decision-making groups," Administrative Science Quarterly, vol. 30, no. 3, pp. 350-372, September 1985.

[17] D. J. Smith, "Situation(al) Awareness (SA) in effective command and control," http://www.smithsrisca.demon.co.uk/situational-awareness. html, accessed Sep 19, 2009.

[18] J. Holley, "Transforming your regional economy through uncertainty and surprise: Learning from complexity science, network theory and the field," in Uncertainty and Surprise in Complex Systems, R. R. McDaniel Jr. and D. J. Driebe, Eds. Springer, 2005, pp. 165-176.

[19] A.-M. Grisogono and A. Ryan, "Operationalising adaptive campaigning," in International Command and Control Research and Technology Symposium, 12th ICCRTS, 2007.

[20] E. D. Pulakos, D. W. Dorsey, and S. S. White, "Adaptability in the workplace: selecting an adaptive workforce," in Understanding Adaptability, A Prerequisite for Effective Performance within Complex Environments, C. S. Burke, L. G. Pierce, and E. Salas, Eds. Elsevier, 2006, pp. 41-71.

[21] K. C. Stagl, C. S. Burke, E. Salas, and L. Pierce, "Team adaptation: realizing team synergy," in Understanding Adaptability, A Prerequisite for Effective Performance within Complex Environments, C. S. Burke, L. G. Pierce, and E. Salas, Eds. Elsevier, 2006, pp. 117-141.

[22] R. P. McGlynn, J. L. Sutton, V. L. Sprague, R. M. Demski, and L. G. Pierce, "Development of a team performance task battery to evaluate performance of the command and control vehicle (c2v) crew," Aberdeen Proving Ground, MD: U.S. Army Research Laboratory, Contract Report, 1997.

[23] K. E. Weick and K. M. Sutcliffe, Managing the Unexpected: Assuring High Performance in an Age of Complexity. Jossey-Bass, 2001.

[24] P. Horn, "Autonomic computing: IBM's perspective on the state of information technology," Manifesto by IBM Research, 2001.

[25] C. S. Burke, K. P. Hess, and E. Salas, "Building the adaptive capacity to lead multi-cultural teams," in Understanding Adaptability, A Prerequisite for Effective Performance within Complex Environments, C. S. Burke, L. G. Pierce, and E. Salas, Eds. Elsevier, 2006, pp. 175-211.

[26] E. Mitleton-Kelly, Complex Systems and Evolutionary Perspectives on Organisations: The Application of Complexity Theory to Organisations. Emerald Group Publishing, 2003.

[27] M. M. Siems, "Legal adaptability in Elbonia," International Journal of Law in Context, vol. 2, no. 4, pp. 393-408, December 2006. 